\title{
Traveltime approximations and parameter estimation for orthorhombic media
}

\author{
Nabil Masmoudi ${ }^{1}$ and Tariq Alkhalifah ${ }^{1}$
}

\begin{abstract}
Building anisotropy models is necessary for seismic modeling and imaging. However, anisotropy estimation is challenging due to the trade-off between inhomogeneity and anisotropy. Luckily, we can estimate the anisotropy parameters if we relate them analytically to traveltimes. Using perturbation theory, we have developed traveltime approximations for orthorhombic media as explicit functions of the anellipticity parameters $\eta_{1}$, $\eta_{2}$, and $\Delta \chi$ in inhomogeneous background media. The parameter $\Delta \chi$ is related to Tsvankin-Thomsen notation and ensures easier computation of traveltimes in the background model. Specifically, our expansion assumes an inhomogeneous ellipsoidal anisotropic background model, which can be obtained from well information and stacking velocity analysis. We have
\end{abstract}

used the Shanks transform to enhance the accuracy of the formulas. A homogeneous medium simplification of the traveltime expansion provided a nonhyperbolic moveout description of the traveltime that was more accurate than other derived approximations. Moreover, the formulation provides a computationally efficient tool to solve the eikonal equation of an orthorhombic medium, without any constraints on the background model complexity. Although, the expansion is based on the factorized representation of the perturbation parameters, smooth variations of these parameters (represented as effective values) provides reasonable results. Thus, this formulation provides a mechanism to estimate the three effective parameters $\eta_{1}, \eta_{2}$, and $\Delta \chi$. We have derived Dix-type formulas for orthorhombic medium to convert the effective parameters to their interval values.

\section{INTRODUCTION}

The quest for more realistic model representation of the earth's subsurface, while maintaining a balance between accuracy and efficiency, has long been a challenge and a quest of research in the geophysical exploration community. Now, we find that an orthorhombic medium is a good enough model to describe many geologic settings especially those that exhibit azimuthal variation behavior (Tsvankin et al., 2010). Such medium describes one or two orthogonal sets of parallel vertical fractures embedded in a transversely isotropic (TI) or a purely isotropic host rock (Wild and Crampin, 1991; Schoenberg and Helbig, 1997; Bakulin et al., 2000). If the sedimentation and fractures are dipping (but orthogonal), this leads to tilted orthorhombic media. Modeling traveltimes satisfying orthorhombic anisotropy can help in many applications such as traveltime tomography, integral-based Kirchhoff imaging, and fracture detection (Bakulin et al., 2000).
First-arrival traveltimes are conventionally evaluated by solving a nonlinear partial differential equation (PDE), known as the eikonal equation. Ray tracing (Červený, 2001) or discretizing the eikonal equation using finite difference (Vidale, 1990; van Trier and Symes, 1991) allow to obtain the traveltimes needed in many imaging applications. Although finite-difference methods are very efficient to solve the eikonal equation of an isotropic medium, using such methods for anisotropic eikonal equations is a challenge. For instance, discretizing the eikonal equation describing a TI or an orthorhombic medium requires, respectively, finding the traveltime solutions of a quartic or a sextic polynomial at each computational step. To alleviate this difficulty, Ma and Alkhalifah (2013), Waheed and Alkhalifah (2013), and Waheed et al. (2015b) suggest computing solutions of the anisotropic eikonal equation by successively solving the simpler elliptic anisotropy eikonal equation, in which the additional nonlinearity of the more complex (i.e., TI) anisotropy is stored in the elliptic coefficients. Ray tracing for P-waves in

\footnotetext{
Manuscript received by the Editor 7 July 2015; revised manuscript received 7 February 2016; published online 30 May 2016.

${ }^{1}$ King Abdullah University of Science and Technology (KAUST), Physical Science and Engineering, Thuwal, Saudi Arabia. E-mail: nabil.masmoudi@kaust. edu.sa; tariq.alkhalifah@kaust.edu.sa.

(C) 2016 Society of Exploration Geophysicists. All rights reserved.
} 
orthorhombic media with varying tilt is proposed (Masmoudi and Pšenčík, 2014; Kainkaryam et al., 2015). Although ray tracing can provide the most energetic traveltime arrivals, it is less robust in the presence of complex structures, and requires interpolation on regular grids.

An orthorhombic medium is described by nine independent stiffness coefficients. However, under the acoustic approximation, only six anisotropy parameters affect the kinematics of P-wave propagation (Tsvankin, 1997). Alkhalifah (2003) proposes a parameterization with the vertical symmetry-plane normal moveout (NMO) velocities $v_{1}$ and $v_{2}$, the vertical velocity $v_{v}$, the anellipticity parameters $\eta_{1}$ and $\eta_{2}$, and the parameter $\delta_{3}$ that we use here. The first and most important step in a successful processing sequence for $\mathrm{P}$-wave data is to estimate the medium parameters. Existing work on orthorhombic traveltime inversion for reflection data has been done mainly through nonhyperbolic moveout description of the traveltimes (Al-Dajani et al., 1998; Xu et al., 2005; Vasconcelos and Tsvankin, 2006). Despite the improvements to handle large offsets, the accuracy of these approximations degrades at far offsets. Using perturbation theory, Alkhalifah (2011) expands the traveltime solution of the eikonal equation of a TI medium, as a function of the anellipticity parameter $\eta$ and proposes a scanning approach to search for this parameter, given a general inhomogeneous background medium with elliptic anisotropy. In addition, Alkhalifah (2012), Stovas and Alkhalifah (2012), and Waheed et al. (2013) use the same idea to derive traveltime approximations for a tilted symmetry axis TI medium. Recently, Alkhalifah (2013) and Masmoudi and Alkhalifah (2014) develop this concept to approximate traveltimes in horizontal transversely isotropic (HTI) media with arbitrary symmetry-axis azimuth $\phi$, and estimate the anisotropy parameter $\eta$ and the azimuthal angle $\phi$.

In this paper, we propose a multiparameter expansion of the traveltimes with respect to the parameters $\eta_{1}, \eta_{2}$, and $\Delta \chi$ with coefficients obtained from solving simple PDE. We derive the analytical solutions of the coefficients of the traveltime expansion, and assess the accuracy of the new approximations with a homogeneous model example. To illustrate the efficiency of our approach, we show examples of traveltime computation in inhomogeneous orthorhombic media, as well as tests of parameter estimation.

\section{ORTHORHOMBIC TRAVELTIME APPROXIMATION}

The eikonal equation describing an orthorhombic medium, under the acoustic assumption, can be written as (Alkhalifah, 2003; Ibanez-Jacome et al., 2014)

$$
\begin{aligned}
& A\left(\frac{\partial \tau}{\partial x}\right)^{2}+B\left(\frac{\partial \tau}{\partial y}\right)^{2}+C\left(\frac{\partial \tau}{\partial z}\right)^{2}+D\left(\frac{\partial \tau}{\partial x}\right)^{2}\left(\frac{\partial \tau}{\partial y}\right)^{2} \\
& \quad+E\left(\frac{\partial \tau}{\partial x}\right)^{2}\left(\frac{\partial \tau}{\partial z}\right)^{2}+F\left(\frac{\partial \tau}{\partial y}\right)^{2}\left(\frac{\partial \tau}{\partial z}\right)^{2} \\
& \quad+G\left(\frac{\partial \tau}{\partial x}\right)^{2}\left(\frac{\partial \tau}{\partial y}\right)^{2}\left(\frac{\partial \tau}{\partial z}\right)^{2}=1
\end{aligned}
$$

where $\tau$ is the traveltime at a grid point with coordinates $(x, y, z)$. In equation 1 , we assume that the symmetry planes of the orthorhombic medium coincide with the coordinate planes. The coefficients $A$, $B, C, D, E, F$, and $G$ are shown below:

$$
\begin{aligned}
A= & v_{1}^{2}\left(1+2 \eta_{1}\right), B=v_{2}^{2}\left(1+2 \eta_{2}\right), C=v_{v}^{2}, \\
D= & v_{1}^{2}\left(1+2 \eta_{1}\right)\left(\left(1+2 \eta_{1}\right) \chi^{2} v_{1}^{2}-\left(1+2 \eta_{2}\right) v_{2}^{2}\right), E=-2 \eta_{1} v_{1}^{2} v_{v}^{2} \\
F= & -2 \eta_{2} v_{2}^{2} v_{v}^{2}, G=-v_{v}^{2} v_{1}^{2}\left(\left(1+2 \eta_{1}\right)^{2} v_{1}^{2} \chi^{2}\right. \\
& \left.-2\left(1+2 \eta_{1}\right) v_{1} v_{2} \chi+v_{2}^{2}\left(1-4 \eta_{1} \eta_{2}\right)\right),
\end{aligned}
$$

where $v_{v}$ is the P-wave vertical velocity, $v_{1}$ and $v_{2}$ are the NMO Pwave velocities for horizontal reflectors in the $[x, z]$ - and $[y, z]$-planes, respectively; $\eta_{1}$ and $\eta_{2}$ are the anellipticity anisotropy parameters in the $[x, z]$ - and $[y, z]$-planes, respectively; and $\chi$ describes the $[x, y]$ horizontal symmetry plane, and it is related to the parameter $\delta_{3}$ (Tsvankin, 1997), through the relation $\chi=\sqrt{1+2 \delta_{3}}$. The parameters $\eta_{1}$ and $\eta_{2}$ can also be expressed in terms of the anisotropy parameters $\epsilon_{1}, \epsilon_{2}, \delta_{1}$, and $\delta_{2}$ as follows:

$$
\eta_{1}=\frac{\epsilon_{1}-\delta_{1}}{1+2 \delta_{1}}, \quad \eta_{2}=\frac{\epsilon_{2}-\delta_{2}}{1+2 \delta_{2}},
$$

with $\epsilon_{1}$ and $\delta_{1}$ are defined in the $[x, z]$ symmetry plane, whereas $\epsilon_{2}$ and $\delta_{2}$ are defined in the $[y, z]$ symmetry plane. The relations between $\delta_{1}, \delta_{2}$ and $v_{1}, v_{2}, v_{v}$ are

$$
v_{1}=v_{v} \sqrt{1+2 \delta_{1}}, \quad v_{2}=v_{v} \sqrt{1+2 \delta_{2}} .
$$

Discretizing equation 1 using finite difference leads to a six-order polynomial (in terms of the traveltime $\tau$ ). Obtaining the roots of such a polynomial is very difficult. On the other hand, solving the eikonal equation for the simple ellipsoidal anisotropic (EA) medium, after a finite-difference discretization, is cheaper (Waheed et al., 2015b). In fact, by setting $\eta_{1}=0, \eta_{2}=0$, and $\chi=\left(v_{2} / v_{1}\right)$, equation 1 reduces to the eikonal equation for an EA medium:

$$
v_{1}^{2}\left(\frac{\partial \tau}{\partial x}\right)^{2}+v_{2}^{2}\left(\frac{\partial \tau}{\partial y}\right)^{2}+v_{v}^{2}\left(\frac{\partial \tau}{\partial z}\right)^{2}=1
$$

Instead of finding the roots of a six-order polynomial (in terms of $\tau$ ), we solve the nonlinear PDE 1 approximately using perturbation theory. By considering $\eta_{1}, \eta_{2}$, and $\Delta \chi=\chi-\chi_{0}$ (where $\chi=$ $\sqrt{1+2 \delta_{3}}$ and $\left.\chi_{0}=\left(v_{2} / v_{1}\right)\right)$ to be constant and small, we expand the traveltime solution $\tau$ (of the eikonal equation 1 ) as a series expansion in terms of $\eta_{1}, \eta_{2}$, and $\Delta \chi$. The velocities $v_{1}, v_{2}$, and $v_{v}$ can be inhomogeneous models, and constitute the background medium, from which the traveltime $\tau$ is perturbed. Due to the small influence that the parameter $\delta_{3}$ has on surface seismic data comparing with $\eta_{1}$ and $\eta_{2}$, only the first-order term $\Delta \chi$ is considered in the traveltime expansion, as shown below:

$$
\begin{aligned}
& \tau(x, y, z)=\tau_{0}(x, y, z)+\tau_{\eta_{1}}(x, y, z) \eta_{1}+\tau_{\eta_{2}}(x, y, z) \eta_{2} \\
& +\tau_{\eta_{1}^{2}}(x, y, z) \eta_{1}^{2}+\tau_{\eta_{2}^{2}}(x, y, z) \eta_{2}^{2}+\tau_{\eta_{1} \eta_{2}}(x, y, z) \eta_{1} \eta_{2} \\
& +\Delta \chi \tau_{\Delta \chi}(x, y, z)
\end{aligned}
$$

We replace equation 6 into the eikonal equation 1 and obtain PDE, which have the following general form:

$$
v_{1}^{2} \frac{\partial \tau_{0}}{\partial x} \frac{\partial \tau_{i}}{\partial x}+v_{2}^{2} \frac{\partial \tau_{0}}{\partial y} \frac{\partial \tau_{i}}{\partial y}+v_{v}^{2} \frac{\partial \tau_{0}}{\partial z} \frac{\partial \tau_{i}}{\partial z}=f_{i}(x, y, z),
$$


a)

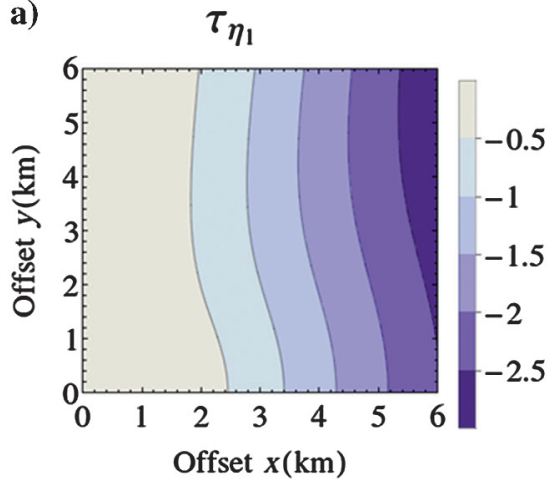

d)

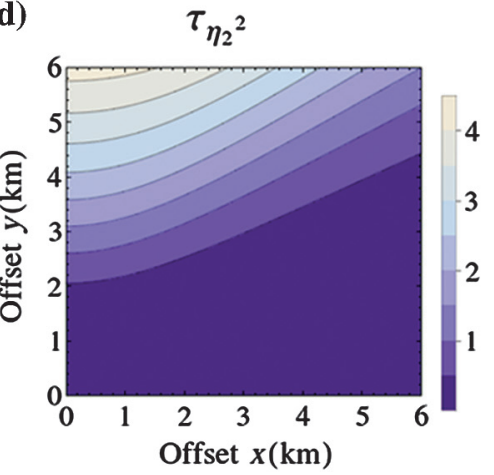

b)

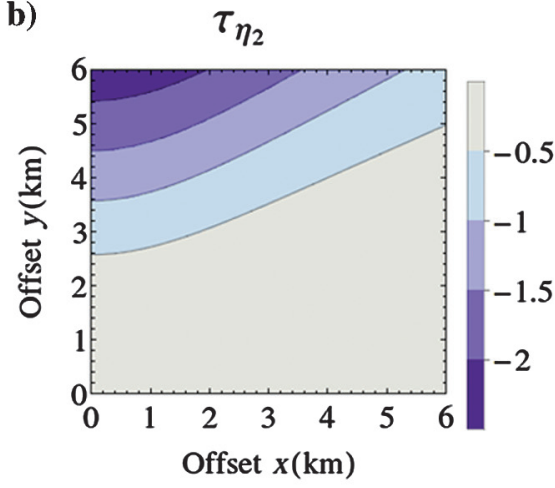

e)

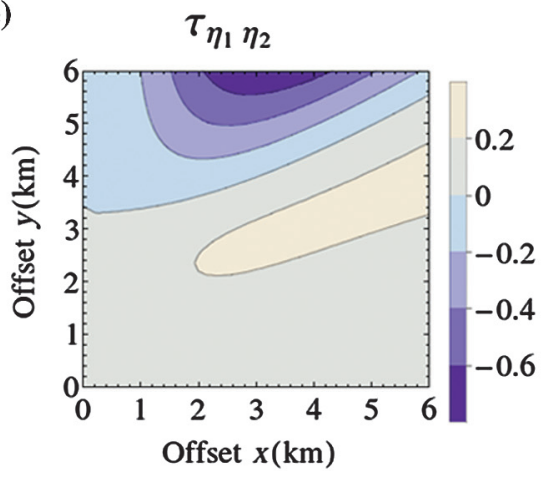

c)

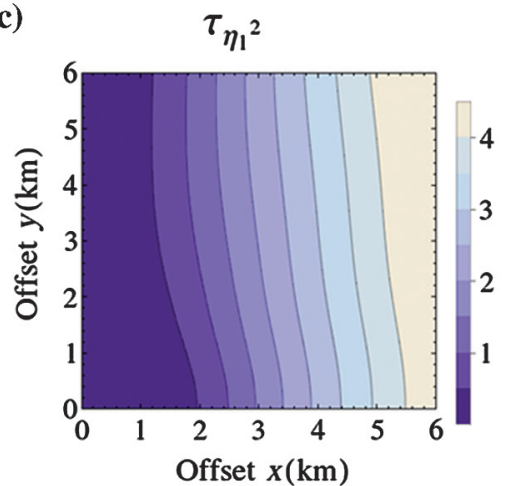

f)

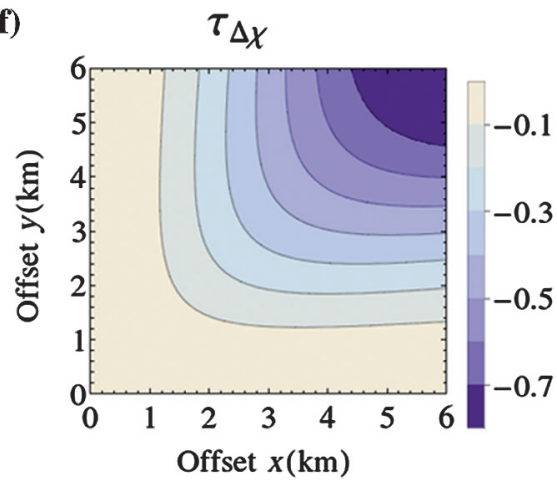

Figure 1. Coefficients of the traveltime expansion 6 as a function of $x$ - and $y$-offsets, for a model with $v_{1}=2, v_{2}=2.1, v_{v}=1.8 \mathrm{~km} / \mathrm{s}$, and a source at $z=2 \mathrm{~km}$ depth $\left(x=0, y=0\right.$, and $z=2 \mathrm{~km}$ ), respectively: (a) $\tau_{\eta_{1}}$, (b) $\tau_{\eta_{2}}$, (c) $\tau_{\eta_{1}^{2}}$, (d) $\tau_{\eta_{2}^{2}}$, (e) $\tau_{\eta_{1} \eta_{2}}$, and (f) $\tau_{\Delta \chi}$.

a)

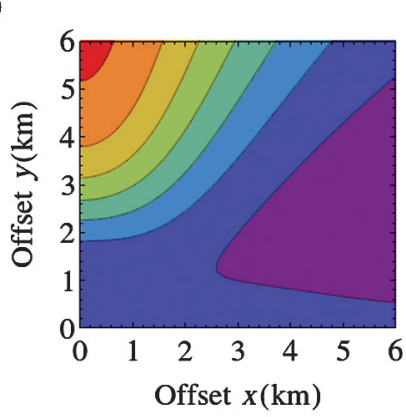

d)

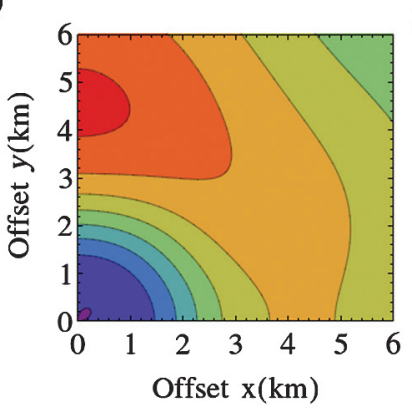

Error(\%)

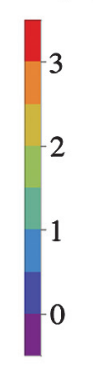

Error(\%)

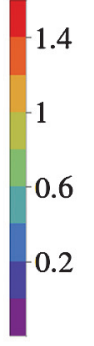

b)

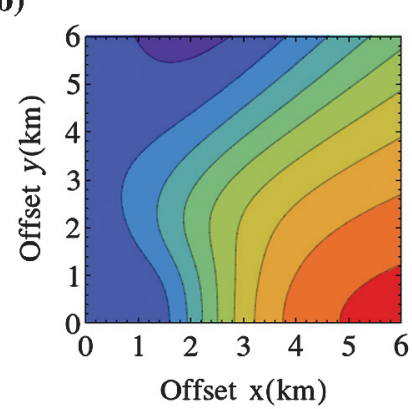

e)

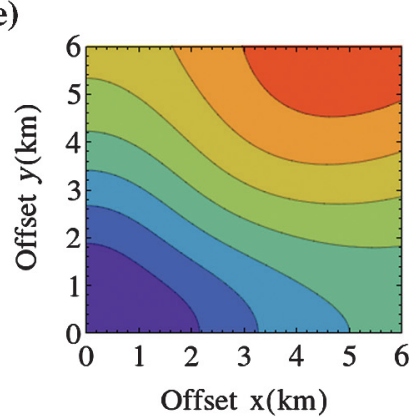

Error $(\%)$

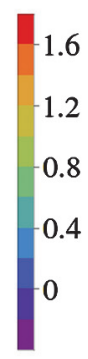

Error(\%)

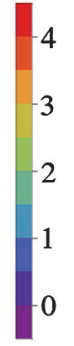

c)

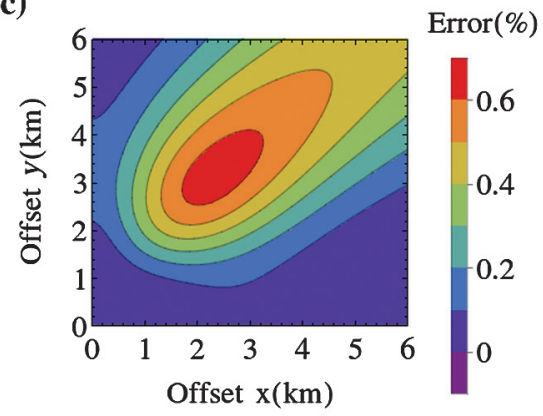

f)

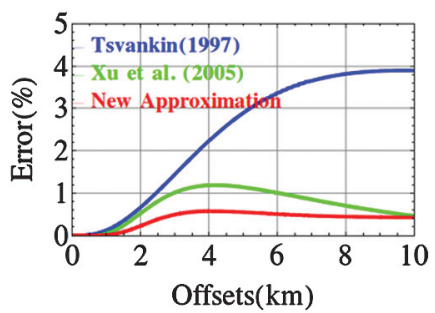

Figure 2. Relative traveltime error as a function of $x$ - and $y$-offsets for a model with $v_{1}=2, v_{2}=2.1, v_{v}=1.8 \mathrm{~km} / \mathrm{s}, \eta_{1}=0.2, \eta_{2}=0.25$, $\delta_{3}=0.15$, and a source at $z=2 \mathrm{~km}$ depth $(x=0, y=0, z=2 \mathrm{~km})$ for (a) expansion 8, (b) expansion 9, (c) approximation 10, (d) Xu et al. (2005) approximation, and (e) Tsvankin (1997) weak anisotropy approximation. (f) A comparison of the errors at the $45^{\circ}$ source-to-receiver azimuth of the Figure $2 \mathrm{c}-2 \mathrm{e}$. 
where the subscript $i$ corresponds to the different coefficients of the expansion $6\left(i=0, \eta_{1}, \eta_{2}, \eta_{1}^{2}, \eta_{2}^{2}, \eta_{1} \eta_{2}, \Delta \chi\right)$. The functions $f_{i}(x, y, z)$ become more complicated for $i$ corresponding to the second-order terms, and they also depend on terms for the first-order and background solutions (see Appendix A). Thus, these equations must be solved in succession starting with the traveltime for the background medium with ellipsoidal anisotropy; then, the coefficients of the first order (i.e., $i=\eta_{1}, i=\eta_{2}, i=\Delta \chi$, etc).

To increase the accuracy of the traveltime approximation 6 , we use two different sequences of Shanks transform (Bender and Orszag, 1978). The idea is to rewrite expansion 6 in two different forms (see Appendix A). In the first form, the expansion is regarded as a polynomial in terms of $\eta_{1}$, whereas in the second form, the expansion is a polynomial in terms of $\eta_{2}$; then, by applying the Shanks transform sequence, one can obtain the following traveltime formulas:

$$
\begin{aligned}
& \tau_{1}(x, y, z)=\tau_{0}(x, y, z)+\tau_{\Delta \chi}(x, y, z) \Delta \chi+\tau_{\eta_{2}}(x, y, z) \eta_{2} \\
& \quad+\tau_{\eta_{2}^{2}}(x, y, z) \eta_{2}^{2} \\
& \quad+\frac{\eta_{1}\left(\tau_{\eta_{1}}(x, y, z)+\tau_{\eta_{1} \eta_{2}}(x, y, z) \eta_{2}\right)^{2}}{\tau_{\eta_{1}}(x, y, z)+\tau_{\eta_{1} \eta_{2}}(x, y, z) \eta_{2}-\eta_{1} \tau_{\eta_{1}^{2}}(x, y, z)}
\end{aligned}
$$
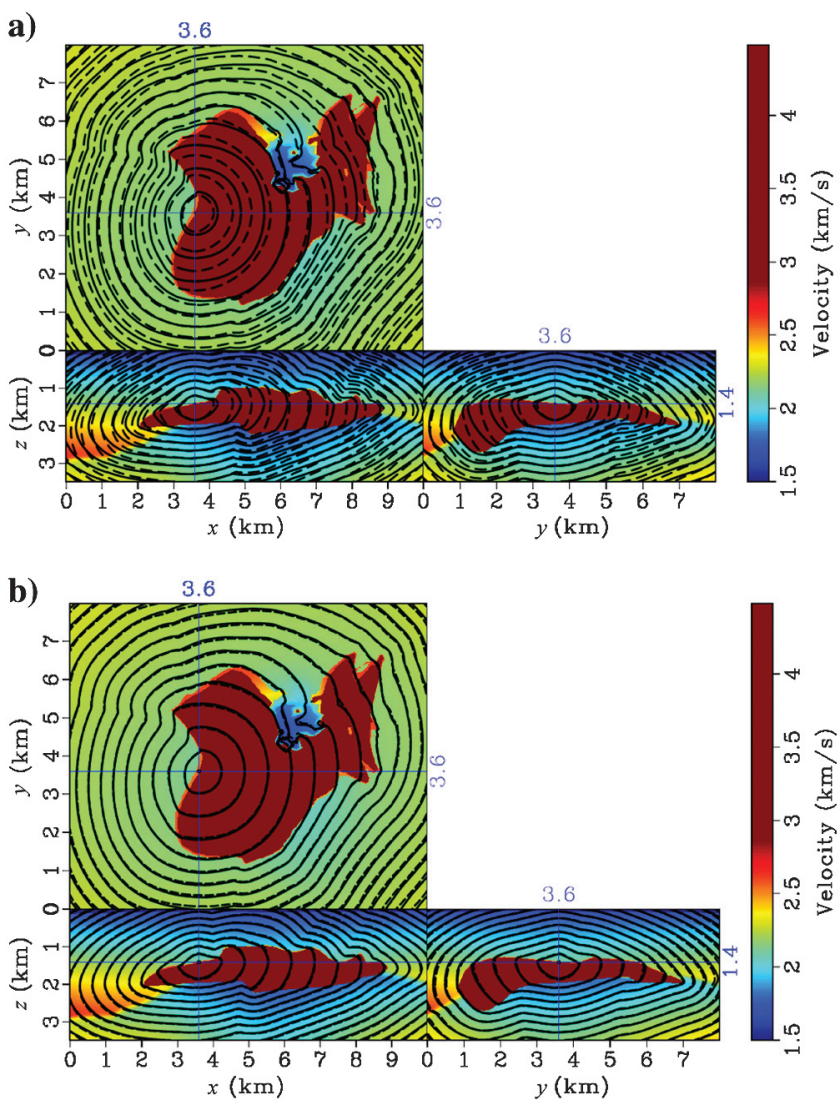

Figure 3. Traveltime contours for SEG/EAGE salt model described by its P-wave vertical velocity and the parameters $\eta_{1}=0.25$, $\eta_{2}=0.2, \delta_{1}=0.2, \delta_{2}=0.15$, and $\delta_{3}=0.1$. Solid contours are the reference traveltimes (Waheed et al., 2015b), and the dashed contours are (a) the ellipsoid background traveltimes (equation 5) and (b) the approximate traveltimes contours (equation 10). In both plots, the P-wave vertical velocity is shown in the background.

$$
\begin{aligned}
& \tau_{2}(x, y, z)=\tau_{0}(x, y, z)+\tau_{\Delta \chi}(x, y, z) \Delta \chi+\tau_{\eta_{1}}(x, y, z) \eta_{1} \\
& \quad+\tau_{\eta_{1}^{2}}(x, y, z) \eta_{1}^{2} \\
& \quad+\frac{\eta_{2}\left(\tau_{\eta_{2}}(x, y, z)+\tau_{\eta_{1} \eta_{2}}(x, y, z) \eta_{1}\right)^{2}}{\tau_{\eta_{2}}(x, y, z)+\tau_{\eta_{1} \eta_{2}}(x, y, z) \eta_{1}-\eta_{2} \tau_{\eta_{2}^{2}}(x, y, z)} .
\end{aligned}
$$

Because the two formulas for $\tau_{1}$ and $\tau_{2}$ are helpful in improving the accuracy of the expansion 6 , using fewer coefficients, respectively, in terms of $\eta_{1}$ and $\eta_{2}$, it is practical to combine them in the following form (Masmoudi and Alkhalifah, 2015b):

$$
\tau(x, y, z)=\tau_{1}(x, y, z) \cos ^{2} \alpha+\tau_{2}(x, y, z) \sin ^{2} \alpha,
$$

where $\alpha$ is the source to receiver azimuth with respect to the $x$ coordinate axis.

\section{ANALYTIC TRAVELTIME APPROXIMATIONS}

Although, the main intention behind developing the traveltime expansion is to estimate the effective parameters $\eta_{1}, \eta_{2}$, and $\Delta \chi$, given a general inhomogeneous background medium, it is interesting to test the accuracy of the new formulas in describing traveltimes for the homogeneous case, especially because most parameter scan-type applications are performed considering an effective homogeneous medium. Considering a homogeneous model with $v_{1}=2, v_{2}=$ 2.1 , and $v_{v}=1.8 \mathrm{~km} / \mathrm{s}$, we compute the analytic coefficients of the traveltime expansion 6, as shown in Appendix B. For this experiment, we consider the one-way traveltimes from a source located at $2 \mathrm{~km}$ depth $(x=0, y=0$, and $z=2 \mathrm{~km})$, and receivers on the surface $(z=0)$. We start by analyzing the behavior of different coefficients because this interpretation gives us insight into the influence of each parameter.

\section{Analyzing the behavior of the traveltime coefficients}

Figure 1 shows the behavior of the coefficients $\tau_{\eta_{1}}, \tau_{\eta_{2}}, \tau_{\eta_{2}^{2}}, \tau_{\eta_{2}^{2}}$, $\tau_{\eta_{1} \eta_{2}}$, and $\tau_{\Delta \chi}$ as a function of the $x$ - and $y$-offsets. Note the increase (in absolute value) of $\tau_{\eta_{1}}$ and $\tau_{\eta_{1}^{2}}$ along the $x$-offset direction and their small variation in the $y$-direction. On the other hand, $\tau_{\eta_{2}}$ and $\tau_{\eta_{2}^{2}}$ variations are mostly associated with the $y$-offsets. This is expected because $\eta_{1}$ is defined in the $[x, z]$-plane, whereas $\eta_{2}$ is defined in the $[y, z]$-plane. Moreover, $\tau_{\eta_{1}}$ decreases with offsets, whereas $\tau_{\eta_{1}^{2}}$ increases with offsets. The same trend applies to $\tau_{\eta_{2}}$ and $\tau_{\eta_{2}^{2}}$. Thus, the coefficients are adjusting the ellipsoid background traveltimes to fit the general nonellipsoid shape of the traveltimes through the first- and second-order terms that stretch the ellipsoid toward a better match. In addition, the values of $\tau_{\Delta \chi}$ are at least three times smaller than $\tau_{\eta_{1}}$ or $\tau_{\eta_{2}}$. This behavior justifies our expansion to only the first order, in terms of $\Delta \chi$. In a nutshell, these coefficients reveal the sensitivity of the traveltime with respect to each parameter; therefore, they can give useful insights in tomographic-based inversions.

\section{Traveltime accuracy for a homogeneous model}

The analytic formulas represent moveout equations describing traveltimes in orthorhombic media as a function of offsets, and can be compared with other traveltime approximations. Xu et al. (2005) extend the P-wave reflection moveout of a vertical TI layer (Tsvankin 
and Thomsen, 1994; Alkhalifah and Tsvankin, 1995) to an orthorhombic layer by considering azimuthally varying parameters as follows:

$$
\begin{aligned}
t^{2}(x)= & t_{0}^{2}(x)+\frac{X^{2}}{V_{\mathrm{NMO}}^{2}(\alpha)} \\
& -\frac{2 \eta(\alpha) X^{4}}{V_{\mathrm{NMO}}^{2}(\alpha)\left(t_{0}^{2} V_{\mathrm{NMO}}^{2}(\alpha)+(1+2 \eta(\alpha)) X^{2}\right)},
\end{aligned}
$$

where $t$ is the reflection traveltime, $t_{0}$ is the two-way zero-offset traveltime, $X$ is the source-receiver offset, and $\eta(\alpha)$ and $V_{\mathrm{NMO}}(\alpha)$ are the azimuthally varying anisotropy parameters defined below:

$$
\begin{aligned}
& V_{\mathrm{NMO}}^{-2}(\alpha)=\frac{\cos ^{2} \alpha}{v_{1}^{2}}+\frac{\sin ^{2} \alpha}{v_{2}^{2}}, \\
& \eta(\alpha)=\eta_{1} \cos ^{2} \alpha+\eta_{2} \sin ^{2} \alpha-\eta_{3} \cos ^{2} \alpha \sin ^{2} \alpha,
\end{aligned}
$$

where $\alpha$ is the azimuth of the source to receiver line with respect to the $x$-axis and $\eta_{3}$ is the anellipticity parameter in the horizontal symmetry plane defined as (Grechka and Tsvankin, 1999)

$$
\eta_{3}=\frac{\epsilon_{2}-\epsilon_{1}-\delta_{3}\left(1+2 \epsilon_{1}\right)}{\left(1+2 \delta_{3}\right)\left(1+2 \epsilon_{1}\right)} .
$$

In addition to Xu et al. (2005) traveltime approximation, we compare our analytic formulas with the traveltimes computed using Tsvankin (1997) weak anisotropy approximation of the phase velocity:

$$
\begin{aligned}
V_{\mathrm{P}}^{2}= & v_{v}^{2}\left(1+2 n_{1}^{4} \epsilon_{1}+2 n_{2}^{4} \epsilon_{2}+2 n_{1}^{2} n_{3}^{2} \delta_{1}\right. \\
& \left.+2 n_{2}^{2} n_{3}^{2} \delta_{2}+2 n_{1}^{2} n_{2}^{2}\left(2 \epsilon_{1}+\delta_{3}\right)\right) .
\end{aligned}
$$

We test the accuracy of the formulas $8-10$ and the two above described approximations (Tsvankin, 1997; Xu et al., 2005) in describing traveltimes for a homogeneous orthorhombic medium. The exact traveltimes are computed using the exact phase and ray velocity for orthorhombic media. We consider the same experiment (source and receiver locations) and background model $\left(v_{1}=2, \quad v_{2}=2.1, \quad v_{v}=1.8 \mathrm{~km} / \mathrm{s}\right)$ described in the introduction of this section. We also consider the anisotropy parameters $\eta_{1}=0.2$, $\eta_{2}=0.25$, and $\delta_{3}=0.15$.

Figure 2a, corresponding to the traveltime formula 8 , shows that the traveltime errors are negligible for a source-to-receiver azimuth smaller than $45^{\circ}$. Figure $2 \mathrm{~b}$, corresponding to the traveltime formula 9 , however, shows that the traveltime errors are generally small for a source-toreceiver azimuth close to $90^{\circ}$. This results from the two different sequences used in the Shanks transform. In $\tau_{1}$, we use a sequence that captures the influence of $\eta_{1}$; therefore, it is very accurate near the $[x, z]$ symmetry plane $\left(\eta_{1}\right.$ is defined in the $[x, z]$-plane), whereas $\tau_{2}$ captures the influence of $\eta_{2}$, thus, achieves better accuracy near the $[y, z]$ symmetry plane $\left(\eta_{2}\right.$ is defined in the $[y, z]$-plane). Therefore, it is practical to combine $\tau_{1}$ and $\tau_{2}$ using equation 10 , which achieves higher traveltime accuracy, as shown in Figure 2c. The azimuthally varying traveltime moveout (Xu et al., 2005) is reasonably accurate (Figure 2d), whereas the weak anisotropy approximation (Tsvankin, 1997) is less accurate in describing the traveltimes (Figure 2e). Figure $2 \mathrm{f}$ compares the errors, at $45^{\circ}$ source-to-receiver azimuth, of different traveltime approximations. Clearly, the new proposed formula 10 is the best performing approximation. Nevertheless, the accuracy of our traveltime approximations tends to decrease for large values of the parameters $\eta_{1}, \eta_{2}$, and $\Delta \chi$; thus, unless higher order coefficients in terms of these parameters are considered, anelliptic approximations such as proposed by Sripanich and Fomel (2015) and Hao and Stovas (2016) are expected to achieve slightly better accuracy. The analytic approximations 8-10 have good accuracy in describing traveltimes for a homogeneous medium; however, they are mainly developed to be used for inhomogeneous orthorhombic media.

\section{TRAVELTIME APPROXIMATION FOR COMPLEX ORTHORHOMBIC MEDIA}

In this section, we test the accuracy of the formula 10 in describing traveltimes for complex inhomogeneous orthorhombic media. a)
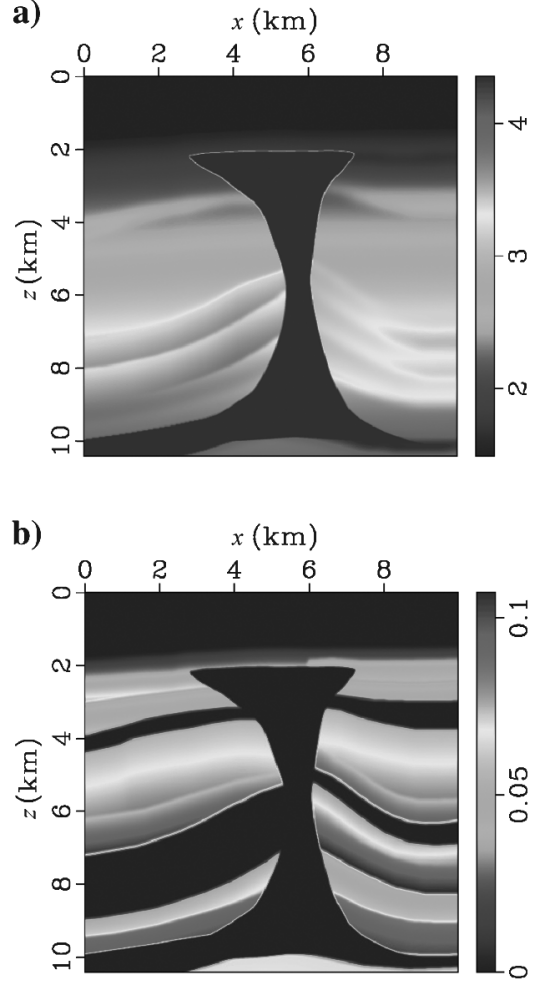

c)

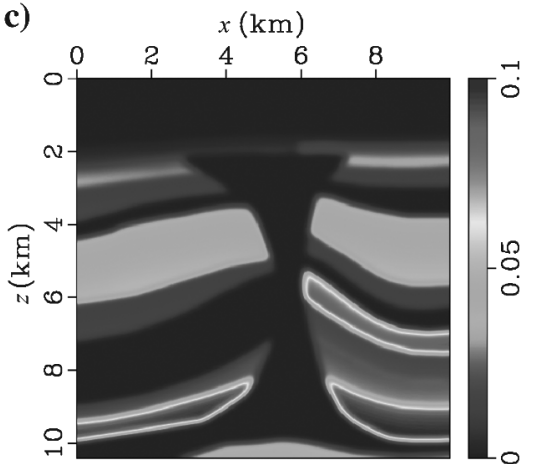

Figure 4. A portion of the 2D TI BP model, specified by (a) the vertical velocity, (b) $\delta_{1}$ and $\eta_{1}$ parameters. (c and d) Two smoothed versions of the original $\eta_{1}$ parameter. The $\mathrm{BP}$ model is extended to orthorhombic by duplicating the 2D models along the $y$-axis and setting the values $\eta_{2}=0.1, \delta_{2}=0.15$, and $\delta_{3}=0.1$. 
To solve numerically the coefficients of the traveltime expansion 6 , we first compute the background traveltimes $\tau_{0}$ using the eikonal equation for a model of ellipsoidal anisotropy (equation 5). Then, we use the background traveltimes to compute the remaining coefficients, which consist of solving linear PDE (see Appendix A). We rely on the fast marching method to solve such equations (Sethian, 1996; Rawlinson and Sambridge, 2004). We show two examples that assess the accuracy of equation 10. The reference traveltime solutions are numerically obtained using the iterative approach of Waheed et al. (2015b).

First, we consider the vertical velocity of the SEG/EAGE salt model, as shown in Figure 3. We extend this model to orthorhombic by specifying five anisotropy parameters $\eta_{1}=0.25, \eta_{2}=0.2$, $\delta_{1}=0.2, \delta_{2}=0.15$, and $\delta_{3}=0.1$. Figure 3 shows the traveltime contours for the reference traveltime solution, the ellipsoid background traveltime, and the traveltime approximation 10 from a

a)

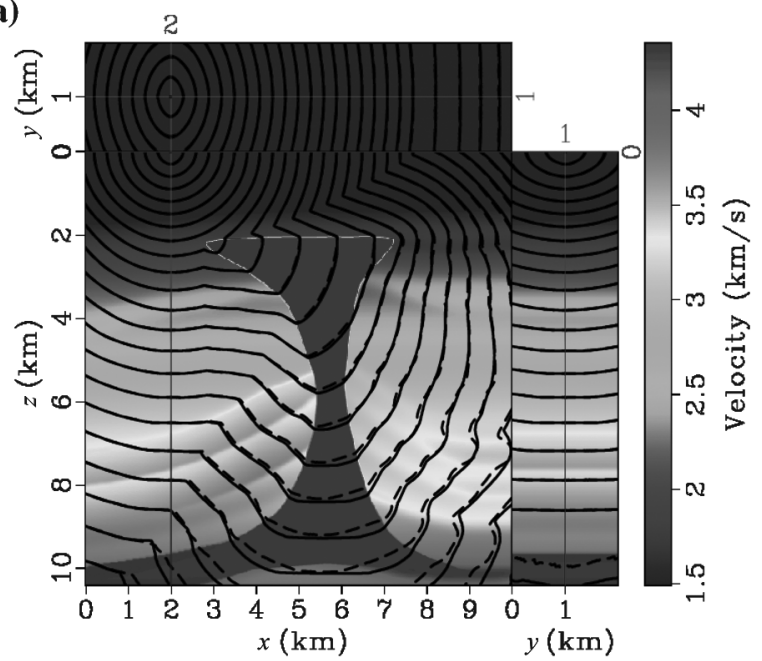

b)

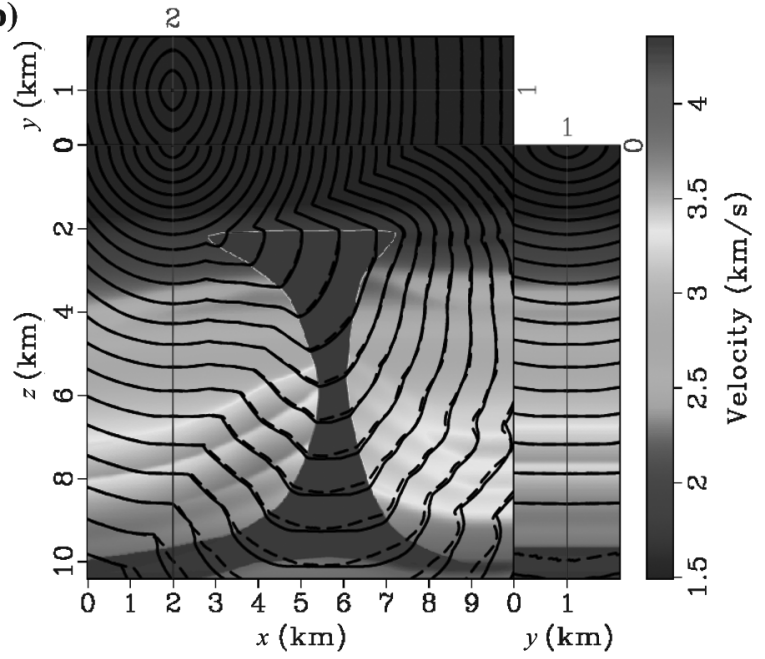

Figure 5. Traveltime contours for the BP model plotted on top of the vertical velocity for two smoothing cases of $\eta_{1}$ parameter. (a) Corresponds to Figure 4c, and (b) corresponds to Figure $4 \mathrm{~d}$. source located at $(x=3.6, y=3.6$, and $z=1.4 \mathrm{~km})$. Though the model is complex (note the difference between the exact and the background traveltime shown in Figure 3a), the traveltime coefficients do a good job in stretching the background traveltimes toward a remarkable fit to the reference traveltimes (Figure $3 b$ ).

The traveltime expansion 6 assumes the parameters $\eta_{1}, \eta_{2}$, and $\Delta \chi$ to be effective and constant. In the second example, we test the possibility of using our approach, even when these parameters have smooth spatial variation. To do so, we consider the parameters $v_{v}$, $\delta_{1}$, and $\eta_{1}$ corresponding to one portion of the BP model, as shown in Figure 4, and we extend them to 3D, by duplicating the same 2D models along the $y$-axis. Also, we choose the constant values $\eta_{2}=0.1, \delta_{2}=0.15$, and $\delta_{3}=0.1$, which make our model orthorhombic. We compare the reference (Waheed et al., 2015b) and the approximate traveltimes (equation 10), computed from a source located at $(x=2, y=1$, and $z=0 \mathrm{~km})$, for two smooth versions of $\eta_{1}$ (see Figure $4 \mathrm{c}$ and $4 \mathrm{~d}$ ). Figure 5 shows the traveltime contours plotted on the top of the vertical velocity. Generally, the approximate traveltime fit the reference traveltime solution. We note, however, a better fit, when a stronger smoothing is applied. For tomography applications resulting in smooth models, the traveltime formulation we propose should be enough accurate. One could also develop a traveltime perturbation equation that allows the spatial variation of the parameters $\eta_{1}, \eta_{2}$, and $\Delta \chi$. The background model in this case, can be ellipsoid and the approach would be similar to the one developed by Waheed et al. (2015a) for a TI medium. Even though this formulation is not suitable for effective anisotropy parameter inversion, it is worthy investigating because it provides more accurate traveltime solutions.

\section{PARAMETER ESTIMATION}

The traveltime approximation 6 is useful in estimating the anisotropy parameters, when a background model is available. Indeed, the coefficients in equation 6 are independent from the parameters $\eta_{1}, \eta_{2}$, and $\Delta \chi$. Therefore, we compute them once, and we use them (in equation 10) to search for best traveltime fit. For example, here,

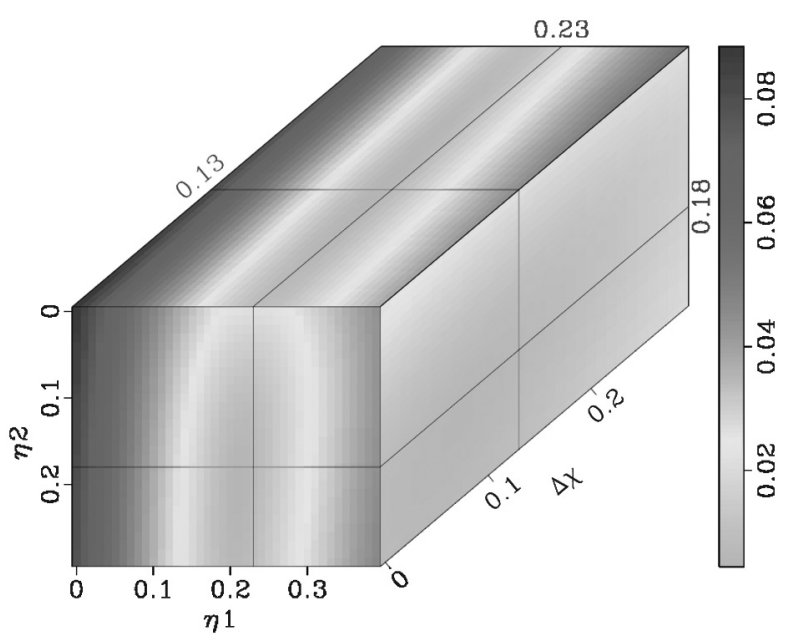

Figure 6. The rms error map (equation 15) for the SEG/EAGE salt model with $\eta_{1}=0.25, \eta_{2}=0.2, \delta_{1}=0.2, \delta_{2}=0.15$, and $\delta_{3}=0.1$. The estimated parameters are $\eta_{1}=0.23, \eta_{2}=0.18$, and $\delta_{3}=0.1$. 
we use the minimum of the root-mean-square (rms) error as a criterion:

$\min _{\eta_{1}, \eta_{2}, \Delta \chi} \operatorname{rms}\left(\eta_{1}, \eta_{2}, \Delta \chi\right)=\min _{\eta_{1}, \eta_{2}, \Delta \chi} \sqrt{\frac{\sum_{r}^{N_{\text {rec }}}\left(\tau_{r}\left(\eta_{1}, \eta_{2}, \Delta \chi\right)-\hat{\tau}_{r}\right)^{2}}{N_{\mathrm{rec}}}}$

to find the best fitting effective parameters $\eta_{1}, \eta_{2}$, and $\Delta \chi$ (Masmoudi and Alkhalifah, 2015a). In equation $15, \tau_{r}\left(\eta_{1}, \eta_{2}, \Delta \chi\right)$ is the traveltime computed using equation $10, \hat{\tau_{r}}$ is the observed traveltime, and $r=1,2, \ldots, N_{\text {rec }}$ with $N_{\text {rec }}$ is the total number of receivers. We show two applications of this approach.

In the first example, we consider the SEG/EAGE salt model (shown in Figure 3), extended to an orthorhombic medium $\left(\eta_{1}=0.25, \eta_{2}=0.2, \delta_{1}=0.2, \delta_{2}=0.15\right.$, and $\left.\delta_{3}=0.1\right)$. The objective here is to estimate the parameters $\eta_{1}, \eta_{2}$, and $\delta_{3}$, given $v_{v}, \delta_{1}$, and $\delta_{2}$. We compute the reference traveltimes (Waheed et al., 2015b) and the coefficients of the traveltime expansion 6 from a source located at $x=3.6, y=3.6$, and $z=1.4 \mathrm{~km}$. Then, we use the criterion 15 , to find the parameters $\eta_{1}, \eta_{2}$, and $\Delta \chi$. In this experiment, we consider only the one way traveltimes from the source to receivers placed on the surface of the model $(z=0 \mathrm{~km})$, with $50 \mathrm{~m}$ spacing along the $x$ - and $y$-axes. Figure 6 shows the rms error (equation 15), as a function of $\eta_{1}, \eta_{2}$, and $\Delta \chi$ (with a sampling of 0.01 for each of the three parameters). Note the elongated shape of the rms error in the plane where $\Delta \chi$ varies. This means that the errors in traveltimes are not very sensitive to this parameter. On the other hand, errors in traveltimes are very sensitive to changes in $\eta_{1}$ and $\eta_{2}$. These observations suggest that $\eta_{1}$ and $\eta_{2}$ are better constrained than $\Delta \chi$. In this example, the minimum rms error yields the best fitting parameters $\eta_{1}=0.23, \eta_{2}=0.18$, and $\Delta \chi=0.13\left(\delta_{3}=0.1\right)$.

In the second example, we estimate the effective parameters of a horizontally layered medium, then, use a Dix-type inversion (see Appendix C) to convert the estimated parameters to their interval values. The size of the layered medium is $4 \times 4 \mathrm{~km}$ laterally and $3 \mathrm{~km}$ in depth, and the thickness of each layer is $1 \mathrm{~km}$. Table 1 shows the interval values of the model. In this experiment, a source is located at $(x=0$, $y=0$, and $z=0 \mathrm{~km}$ ), and receivers are on the surface $(z=0 \mathrm{~km})$ with $50 \mathrm{~m}$ spacing along the $x$ - and $y$-axes. We consider the two-way traveltimes $\tau_{i}$ (the subscript $i$ refers to one of the layers, $i=1,2,3$ ), as shown below:

$$
\tau_{i}=\tau_{i_{0}}+\tau_{s_{i}},
$$

where $\tau_{i_{0}}$ is the zero-offset traveltime and $\tau_{s_{i}}$ is the traveltime from a virtual source located at ( $x=0, y=0$, and $z=d_{i}$ ), where $d_{i}$ is the depth of the $i$ th layer. We compute the coefficients of the traveltime expansion 6 as well as the reference traveltimes (Waheed et al., 2015b), and we use the criterion 15 to search for the effective parameters $\eta_{1}, \eta_{2}$, and $\Delta \chi$ corresponding to each layer. Figure 7 shows the rms error maps associated with each layer, as a function of $\eta_{1}, \eta_{2}$, and model. inversion.
$\Delta \chi$. The minima of these maps give the best effective parameters. We convert these parameters to their interval values (see Table 2) using a layer stripping algorithm based on the relations given in Appendix C. We notice from Table 2 that the errors increase with depth for $\eta_{1}$ and $\eta_{2}$, which is a general behavior of any layer stripping method. However, errors in $\delta_{3}$ in this example do not follow this general trend. Because $\delta_{3}$ has small influence on the traveltimes, its interpretation may be ambiguous.

\section{DISCUSSION}

The proposed traveltime perturbation is a short-cut that provides efficiently computed traveltimes for various models. In many applications, such as parameter estimation or traveltime tomography, the cost saving associated with traveltime computation is significant. Errors in the background model cause inaccuracies when estimating the perturbation parameters. However, like many sequential inversion approaches, we can use these parameters to improve the convergence of a full-waveform inversion, and then use the resulting background model to improve the estimation of the parameters. Moreover, in the case the symmetry planes of the orthorhombic medium do not coincide with the coordinate planes, one could incorporate such variations into the traveltimes describing the ellipsoid background medium, by applying a proper rotation matrix in equation 5 . If the orientation of the symmetry planes is not accurately known, one might attempt to use the same perturbation method presented in this paper to estimate these angular variations, similarly to the approach described in Alkhalifah (2013) and Masmoudi and Alkhalifah (2014) for a HTI medium. However, due to the number of parameters needed to be estimated, application of this approach to orthorhombic medium is more challenging.

Table 1. Interval values of parameters of the horizontally layered orthorhombic

\begin{tabular}{lcccclll} 
Layer & Depth & $v_{v}(\mathrm{~km})$ & $v_{1}(\mathrm{~km} / \mathrm{s})$ & $v_{2}(\mathrm{~km} / \mathrm{s})$ & $\eta_{1}$ & \multicolumn{1}{l}{$\eta_{2}$} & $\delta_{3}$ \\
\hline 1 & 1 & 1.8 & 1.9 & 2 & 0.1 & 0.05 & 0.1 \\
2 & 2 & 1.9 & 2 & 2.1 & 0.15 & 0.1 & 0.15 \\
3 & 3 & 2 & 2.1 & 2.2 & 0.2 & 0.15 & 0.2 \\
\hline
\end{tabular}

Table 2. Effective parameters estimated and errors in interval values after Dix

Layer Effective parameters $\quad$ Errors in interval parameters estimated

\begin{tabular}{lllllll} 
& $\eta_{1}$ & $\eta_{2}$ & $\Delta \chi$ & $\eta_{1}$ & $\eta_{2}$ & $\delta_{3}$ \\
\hline 1 & 0.1 & 0.05 & 0.07 & 0 & 0 & 0.03 \\
2 & 0.12 & 0.07 & 0.08 & 0.014 & 0.014 & 0.003 \\
3 & 0.14 & 0.09 & 0.11 & 0.032 & 0.03 & 0.022 \\
\hline
\end{tabular}



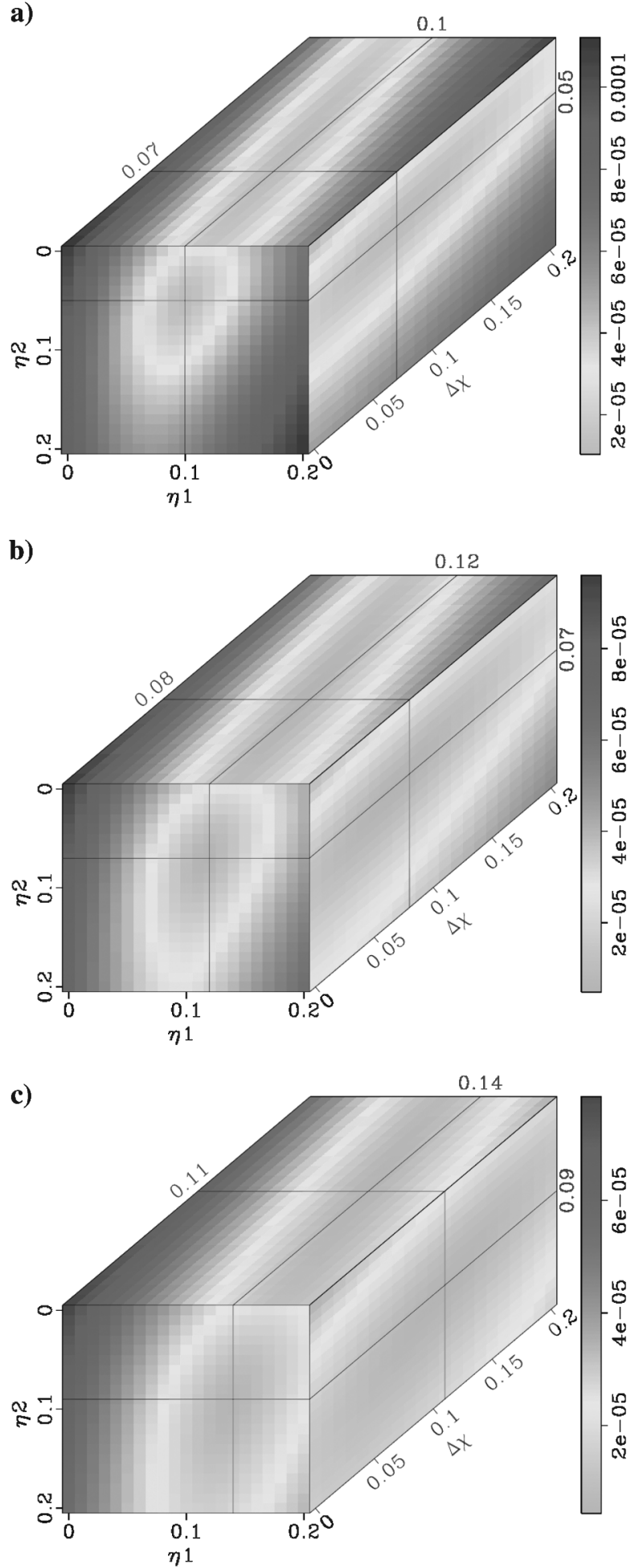

Figure 7. The rms error maps (equation 15) for the layered orthorhombic model (see Table 1), for (a) the upper, (b) middle, and (c) bottom layers.

\section{CONCLUSION}

By expanding the traveltime solution of the eikonal equation describing an orthorhombic medium in a power series in terms of $\eta_{1}$, $\eta_{2}$, and $\Delta \chi$, we have developed new traveltime approximations for orthorhombic media. The analytic representation of the new formulas provides accurate traveltimes description for a homogeneous medium. The numerical examples show the usefulness of the approach in estimating the anisotropy parameters, given an inhomogeneous background model. For an orthorhombic medium with varying symmetry planes orientation, a simple coordinate transformation can be applied to the background traveltime computation if such angular variations are developed.

\section{ACKNOWLEDGMENTS}

We thank KAUST for financial support. We thank A. Stovas for many useful discussions. We also thank U. bin Waheed for his help in accessing his orthorhombic traveltime calculation. We are also grateful to I. Ravve, N. Belayouni, I. Pšenčík, and Y. Luo for their critical and helpful reviews of the paper.

\section{APPENDIX A}

\section{THE TRAVELTIME EXPANSION COEFFICIENTS}

To derive the coefficients of the traveltime expansion in terms of the anisotropy parameters $\eta_{1}, \eta_{2}$, and $\Delta \chi$, we insert the traveltime expansion 6 into the eikonal equation 1 . This yields a complicated formula, but by setting $\eta_{1}=0, \eta_{2}=0$, and $\Delta \chi=0$, one should obtain the coefficient of the zeroth-order term in this form

$$
v_{1}^{2}\left(\frac{\partial \tau_{0}}{\partial x}\right)^{2}+v_{2}^{2}\left(\frac{\partial \tau_{0}}{\partial y}\right)^{2}+v_{v}^{2}\left(\frac{\partial \tau_{0}}{\partial z}\right)^{2}=1,
$$

which is the eikonal equation for ellipsoidal anisotropy. By equating the coefficients of the powers of the independent parameters $\eta_{1}, \eta_{2}$, and $\Delta \chi$ in succession starting with the first power of the parameters, we obtain linear PDE in terms of $\tau_{i}\left(i=\eta_{1}, \eta_{2}, \eta_{1}^{2}, \eta_{2}^{2}, \eta_{1} \eta_{2}, \Delta \chi\right)$ in this general form

$$
v_{1}^{2} \frac{\partial \tau_{0}}{\partial x} \frac{\partial \tau_{i}}{\partial x}+v_{2}^{2} \frac{\partial \tau_{0}}{\partial y} \frac{\partial \tau_{i}}{\partial y}+v_{v}^{2} \frac{\partial \tau_{0}}{\partial z} \frac{\partial \tau_{i}}{\partial z}=f_{i}(x, y, z),
$$

where $f_{i}$ is the source term. We get the function $f_{\eta_{1}}$ for the coefficient of first power in $\eta_{1}$ as shown below:

$$
\begin{aligned}
f_{\eta_{1}} & =v_{1}^{2}\left(v_{v}^{2}\left(\frac{\partial \tau_{0}}{\partial z}\right)^{2}\left(\frac{\partial \tau_{0}}{\partial x}\right)^{2}\right. \\
& \left.-v_{2}^{2}\left(\frac{\partial \tau_{0}}{\partial y}\right)^{2}\left(\frac{\partial \tau_{0}}{\partial x}\right)^{2}-\left(\frac{\partial \tau_{0}}{\partial x}\right)^{2}\right) .
\end{aligned}
$$

Moreover, the source term $f_{\eta_{2}}$ for the coefficient of first power in $\eta_{2}$ has this expression

$$
\begin{aligned}
f_{\eta_{2}} & =v_{2}^{2}\left(v_{v}^{2}\left(\frac{\partial \tau_{0}}{\partial z}\right)^{2}\left(\frac{\partial \tau_{0}}{\partial y}\right)^{2}\right. \\
& \left.+v_{1}^{2}\left(\frac{\partial \tau_{0}}{\partial y}\right)^{2}\left(\frac{\partial \tau_{0}}{\partial x}\right)^{2}-\left(\frac{\partial \tau_{0}}{\partial y}\right)^{2}\right) .
\end{aligned}
$$

We obtain the source term $f_{\Delta \chi}$ for the coefficient of first power in $\Delta \chi$ as follows: 


$$
f_{\Delta \chi}=-v_{1}^{3} v_{2}\left(\frac{\partial \tau_{0}}{\partial y}\right)^{2}\left(\frac{\partial \tau_{0}}{\partial x}\right)^{2}
$$

The source function $f_{\eta_{1}^{2}}$ for the coefficient of second power in terms of $\eta_{1}$ and zeroth power in $\eta_{2}$ and $\Delta \chi$ depends on the background traveltime solution $\tau_{0}$ as well as on the coefficient of first power in $\eta_{1}$

$$
\begin{aligned}
f_{\eta_{1}^{2}}= & -\frac{1}{2}\left(v_{v}^{2}\left(\frac{\partial \tau_{\eta_{1}}}{\partial z}\right)^{2}+v_{1}^{2}\left(\frac{\partial \tau_{\eta_{1}}}{\partial x}\right)^{2}+v_{2}^{2}\left(\frac{\partial \tau_{\eta_{1}}}{\partial y}\right)^{2}\right) \\
& +2 v_{1}^{2}\left(\frac{\partial \tau_{0}}{\partial x}\right)^{2}\left(-v_{2}^{2} \frac{\partial \tau_{0}}{\partial y} \frac{\partial \tau_{\eta_{1}}}{\partial y}+v_{v}^{2} \frac{\partial \tau_{0}}{\partial z} \frac{\partial \tau_{\eta_{1}}}{\partial z}\right) \\
& +2 v_{1}^{2} \frac{\partial \tau_{0}}{\partial x} \frac{\partial \tau_{\eta_{1}}}{\partial x}\left(v_{v}^{2}\left(\frac{\partial \tau_{0}}{\partial z}\right)^{2}-v_{2}^{2}\left(\frac{\partial \tau_{0}}{\partial y}\right)^{2}-1\right) \\
& +2 v_{1}^{2} v_{2}^{2}\left(\frac{\partial \tau_{0}}{\partial x}\right)^{2}\left(\frac{\partial \tau_{0}}{\partial y}\right)^{2}\left(v_{v}^{2}\left(\frac{\partial \tau_{0}}{\partial z}\right)^{2}-1\right) .
\end{aligned}
$$

The source function for the coefficient of second power in $\eta_{2}$ and zeroth power in $\eta_{2}$ and $\Delta \chi$ has this form

$$
\begin{aligned}
f_{\eta_{2}^{2}}= & -\frac{1}{2}\left(v_{v}^{2}\left(\frac{\partial \tau_{\eta_{2}}}{\partial z}\right)^{2}+v_{1}^{2}\left(\frac{\partial \tau_{\eta_{2}}}{\partial x}\right)^{2}+v_{2}^{2}\left(\frac{\partial \tau_{\eta_{2}}}{\partial y}\right)^{2}\right) \\
& +2 v_{2}^{2} \frac{\partial \tau_{0}}{\partial y} \frac{\partial \tau_{\eta_{2}}}{\partial y}\left(v_{v}^{2}\left(\frac{\partial \tau_{0}}{\partial z}\right)^{2}+v_{1}^{2}\left(\frac{\partial \tau_{0}}{\partial x}\right)^{2}-1\right) \\
& +2 v_{2}^{2}\left(\frac{\partial \tau_{0}}{\partial y}\right)^{2}\left(v_{1}^{2} \frac{\partial \tau_{0}}{\partial x} \frac{\partial \tau_{\eta_{2}}}{\partial x}+v_{v}^{2} \frac{\partial \tau_{0}}{\partial z} \frac{\partial \tau_{\eta_{2}}}{\partial z}\right),
\end{aligned}
$$

which depends on the background traveltime $\tau_{0}$ as well as on the coefficient of first power in $\eta_{2}$. Finally, the function $f_{\eta_{1} \eta_{1}}$ for the coefficient of first power in $\eta_{1}$ and $\eta_{2}$ as well as zeroth power in $\Delta \chi$ results in the following relation:

$$
\begin{aligned}
f_{\eta_{1} \eta_{2}}= & 2 v_{1}^{2}\left(\frac{\partial \tau_{0}}{\partial x}\right)^{2}\left(v_{2}^{2} \frac{\partial \tau_{0}}{\partial y} \frac{\partial \tau_{\eta_{1}}}{\partial y}-v_{2}^{2} \frac{\partial \tau_{0}}{\partial y} \frac{\partial \tau_{\eta_{2}}}{\partial y}+v_{v}^{2} \frac{\partial \tau_{0}}{\partial z} \frac{\partial \tau_{\eta_{2}}}{\partial z}\right) \\
& +2 v_{2}^{2}\left(\frac{\partial \tau_{0}}{\partial y}\right)^{2}\left(v_{1}^{2} \frac{\partial \tau_{0}}{\partial x} \frac{\partial \tau_{\eta_{1}}}{\partial x}-v_{1}^{2} \frac{\partial \tau_{0}}{\partial x} \frac{\partial \tau_{\eta_{2}}}{\partial x}+v_{v}^{2} \frac{\partial \tau_{0}}{\partial z} \frac{\partial \tau_{\eta_{1}}}{\partial z}\right) \\
& +2 v_{1}^{2} v_{2}^{2}\left(\frac{\partial \tau_{0}}{\partial x}\right)^{2}\left(\frac{\partial \tau_{0}}{\partial y}\right)^{2}\left(1-v_{v}^{2}\left(\frac{\partial \tau_{0}}{\partial z}\right)^{2}\right) \\
& -v_{1}^{2} \frac{\partial \tau_{\eta_{2}}}{\partial x}\left(2 \frac{\partial \tau_{0}}{\partial x}+\frac{\partial \tau_{\eta_{1}}}{\partial x}\right)-v_{2}^{2} \frac{\partial \tau_{\eta_{1}}}{\partial y}\left(2 \frac{\partial \tau_{0}}{\partial y}+\frac{\partial \tau_{\eta_{2}}}{\partial y}\right) \\
& -v_{v}^{2} \frac{\partial \tau_{\eta_{1}}}{\partial z} \frac{\partial \tau_{\eta_{2}}}{\partial z} \\
& +2 v_{v}^{2}\left(\frac{\partial \tau_{0}}{\partial z}\right)^{2}\left(v_{1}^{2} \frac{\partial \tau_{0}}{\partial x} \frac{\partial \tau_{\eta_{2}}}{\partial x}+v_{2}^{2} \frac{\partial \tau_{0}}{\partial y} \frac{\partial \tau_{\eta_{1}}}{\partial y}\right) .
\end{aligned}
$$

Though equations A-6 to A-8 seem complicated, many of the variables of the source functions can be evaluated during the evaluation of equation A-2 (with the source terms A-3 and A-4) in a fashion that will not add much to the cost.

We use Shanks transform (Bender and Orszag, 1978) to improve the accuracy of the traveltime expansion 6. Depending on the considered coefficients defined in the Shanks transform sequence, we can obtain different formulas. First, we define

$$
\begin{aligned}
& A_{0}=\tau_{0}+\tau_{\eta_{2}} \eta_{2}+\tau_{\eta_{2}^{2}} \eta_{2}^{2}+\tau_{\Delta \chi} \Delta \chi \\
& A_{1}=A_{0}+\left(\tau_{\eta_{1}}+\tau_{\eta_{1} \eta_{2}} \eta_{2}\right) \eta_{1}, \\
& A_{2}=A_{1}+\tau_{\eta_{1}^{2}} \eta_{1}^{2}
\end{aligned}
$$

which represent the coefficients of Shanks transform when equation 6 is regarded as an expansion in terms of $\eta_{1}$. Then, by applying Shanks transform, we obtain the following expression:

$$
\begin{aligned}
\tau_{1}(x, y, z) & =\frac{A_{0} A_{2}-A_{1}^{2}}{A_{0}-2 A_{1}+A_{2}}, \\
& =\tau_{0}(x, y, z)+\tau_{\Delta \chi}(x, y, z) \Delta \chi+\tau_{\eta_{2}}(x, y, z) \eta_{2} \\
& +\tau_{\eta_{2}^{2}}(x, y, z) \eta_{2}^{2} \\
& +\frac{\eta_{1}\left(\tau_{\eta_{1}}(x, y, z)+\tau_{\eta_{1} \eta_{2}}(x, y, z) \eta_{2}\right)^{2}}{\tau_{\eta_{1}}(x, y, z)+\tau_{\eta_{1} \eta_{2}}(x, y, z) \eta_{2}-\eta_{1} \tau_{\eta_{1}^{2}}(x, y, z)}
\end{aligned}
$$

Similarly to equation A-10, another approximation can be obtained by defining first

$$
\begin{aligned}
& B_{0}=\tau_{0}+\tau_{\eta_{1}} \eta_{1}+\tau_{\eta_{1}^{2}} \eta_{1}^{2}+\tau_{\Delta \chi} \Delta \chi, \\
& B_{1}=B_{0}+\left(\tau_{\eta_{2}}+\tau_{\eta_{1} \eta_{2}} \eta_{1}\right) \eta_{2}, \\
& B_{2}=B_{1}+\tau_{\eta_{2}^{2}} \eta_{2}^{2},
\end{aligned}
$$

which represent the coefficients of Shanks transform when equation 6 is regarded as an expansion in terms of $\eta_{2}$; then, we obtain the following traveltime approximation:

$$
\begin{aligned}
& \tau_{2}(x, y, z)=\frac{B_{0} B_{2}-B_{1}^{2}}{B_{0}-2 B_{1}+B_{2}}, \\
& =\tau_{0}(x, y, z)+\tau_{\Delta \chi}(x, y, z) \Delta \chi+\tau_{\eta_{1}}(x, y, z) \eta_{1}+\tau_{\eta_{1}^{2}}(x, y, z) \eta_{1}^{2} \\
& +\frac{\eta_{2}\left(\tau_{\eta_{2}}(x, y, z)+\tau_{\eta_{1} \eta_{2}}(x, y, z) \eta_{1}\right)^{2}}{\tau_{\eta_{2}}(x, y, z)+\tau_{\eta_{1} \eta_{2}}(x, y, z) \eta_{1}-\eta_{2} \tau_{\eta_{2}^{2}}(x, y, z)}
\end{aligned}
$$

Because $\tau_{1}$ is more accurate near the $[x, z]$ symmetry plane, whereas $\tau_{2}$ has better accuracy near the $[y, z]$ symmetry plane, it is practical to combine them both in the following form:

$$
\tau(x, y, z)=\tau_{1}(x, y, z) \cos ^{2} \alpha+\tau_{2}(x, y, z) \sin ^{2} \alpha,
$$

where $\alpha$ is the source-to-receiver azimuth with respect to the $x$-coordinate axis.

\section{APPENDIX B}

\section{THE HOMOGENEOUS MEDIUM CASE}

To develop the analytic solutions for the coefficients of the traveltime expansion 6, we start with a background traveltime solution given by 


$$
\tau_{0}=\sqrt{\frac{x^{2}}{v_{1}^{2}}+\frac{y^{2}}{v_{2}^{2}}+\frac{z^{2}}{v_{v}^{2}}}
$$

which satisfies the eikonal equation A-1 of ellipsoid anisotropy. Using equation B-1, we evaluate $\partial \tau_{0} / \partial x, \partial \tau_{0} / \partial y$, and $\partial \tau_{0} / \partial z$, and use them in solving the linear PDE A-2 (with the source term given by equations A-3 to A-5). We obtain the coefficients $\tau_{\eta_{1}}, \tau_{\eta_{2}}$, and $\tau_{\Delta \chi}$ as follows:

$$
\begin{gathered}
\tau_{\eta_{1}}=-\frac{\tau_{0} v_{v}^{4}}{\lambda}\left(v_{2}^{4} x^{4}+2 v_{1}^{2} v_{2}^{2} x^{2} y^{2}\right), \\
\tau_{\eta_{2}}=-\frac{\tau_{0} v_{v}^{4}}{\lambda} v_{1}^{4} y^{4} \\
\tau_{\Delta \chi}=-\frac{\tau_{0} v_{v}^{4}}{\lambda} v_{1}^{3} v_{2} x^{2} y^{2},
\end{gathered}
$$

where $\lambda$ has the form shown below:

$$
\lambda=\left(v_{1}^{2} v_{2}^{2} z^{2}+v_{1}^{2} v_{v}^{2} y^{2}+v_{2}^{2} v_{v}^{2} x^{2}\right)^{2} .
$$

Then, using equation B-2 we evaluate $\partial \tau_{\eta_{1}} / \partial x, \partial \tau_{\eta_{1}} / \partial y$, and $\partial \tau_{\eta_{1}} / \partial z$, and we use them to solve the PDE A-2 (with the source term A-6) to get $\tau_{\eta_{1}^{2}}$

$$
\begin{aligned}
\tau_{\eta_{1}^{2}}= & \frac{3 v_{v}^{4} x^{2}}{2 \lambda^{3 / 2} \tau_{0} v_{1}^{2}}\left(v_{v}^{2}\left(4 v_{1}^{6} y^{6}+4 v_{1}^{4} v_{2}^{2} x^{2} y^{4}+4 v_{1}^{2} v_{2}^{4} x^{4} y^{2}+v_{2}^{6} x^{6}\right)\right. \\
& \left.+4 v_{1}^{2} v_{2}^{2} z^{2}\left(v_{1}^{4} y^{4}+3 v_{1}^{2} v_{2}^{2} x^{2} y^{2}+v_{2}^{4} x^{4}\right)\right) .
\end{aligned}
$$

Similarly, we use equation B-3 to compute $\partial \tau_{\eta_{2}} / \partial x, \partial \tau_{\eta_{2}} / \partial y$, and $\partial \tau_{\eta_{2}} / \partial z$, and then we solve equation A-2 (with the source term A-7) to obtain $\tau_{\eta_{2}^{2}}$

$$
\tau_{\eta_{2}^{2}}=\frac{3 v_{1}^{4} v_{v}^{4} y^{6}}{2 \lambda^{3 / 2} \tau_{0} v_{2}^{2}}\left(4 v_{2}^{2}\left(v_{1}^{2} z^{2}+v_{v}^{2} x^{2}\right)+v_{1}^{2} v_{v}^{2} y^{2}\right)
$$

Finally, we use the previously computed partial derivatives of $\tau_{\eta_{1}}$ and $\tau_{\eta_{2}}$ to solve equation A-2 (with the source term A-8) and obtain the following expression for $\tau_{\eta_{1} \eta_{2}}$ :

$$
\tau_{\eta_{1} \eta_{2}}=\frac{3 v_{1}^{2} v_{v}^{4} x^{2} y^{4}}{\lambda^{3 / 2} \tau_{0}}\left(v_{2}^{2}\left(4 v_{1}^{2} z^{2}+v_{v}^{2} x^{2}\right)-2 v_{1}^{2} v_{v}^{2} y^{2}\right)
$$

\section{APPENDIX C}

\section{DIX-TYPE FORMULAS FOR ORTHORHOMBIC MEDIA}

The eikonal equation 1 can be written in terms of the components $\left(p_{x}, p_{y}, p_{z}\right)$ of the slowness vector as shown below: $p_{z}=\frac{\sqrt{1-p_{y}^{2} V_{2}^{2}-p_{x}^{2} V_{1}^{2}\left(1+p_{y}^{2}\left(\chi^{2} v_{1}^{2}-v_{2}^{2}+2 \chi^{2} v_{1}^{2} \eta_{1}-2 v_{2}^{2} \eta_{2}\right)\right)}}{v_{v} \sqrt{1-2 p_{y}^{2} v_{2}^{2} \eta_{2}-p_{x}^{2} v_{1}^{2}\left(2 \eta_{1}+p_{y}^{2}\left(\chi^{2} v_{1}^{2}\left(1+2 \eta_{1}\right)^{2}-2 \chi v_{1} v_{2}\left(1+2 \eta_{1}\right)+v_{2}^{2}\left(1-4 \eta_{1} \eta_{2}\right)\right)\right)}}$.

Here, $V_{1}$ and $V_{2}$ are the horizontal velocities defined as

$$
\begin{aligned}
& V_{1}=v_{1} \sqrt{1+2 \eta_{1}}, \\
& V_{2}=v_{2} \sqrt{1+2 \eta_{2}} .
\end{aligned}
$$

We expand the vertical slowness $p_{z}$ around zero-offset ray $\left(p_{x}=0\right.$ and $p_{y}=0$ ) up to the fourth order as follows:

$$
p_{z}=a_{0}+a_{x^{2}} p_{x}^{2}+a_{y^{2}} p_{y}^{2}+a_{x^{4}} p_{x}^{4}+a_{y^{4}} p_{y}^{4}+a_{x^{2} y^{2}} p_{x}^{2} p_{y}^{2},
$$

with only coefficients corresponding to even power in $p_{x}$ and $p_{y}$ that are nonzero. We obtain the expressions of $a_{0}, a_{x^{2}}, a_{y^{2}}, a_{x^{4}}, a_{y^{4}}, a_{x^{2} y^{2}}$ shown below:

$$
\begin{aligned}
a_{0} & =\frac{1}{v_{v}} \\
a_{x^{2}} & =-\frac{v_{1}^{2}}{2 v_{v}}, \\
a_{y^{2}} & =-\frac{v_{2}^{2}}{2 v_{v}}, \\
a_{x^{4}} & =-\frac{\left(1+8 \eta_{1}\right) v_{1}^{4}}{8 v_{v}}, \\
a_{y^{4}} & =-\frac{\left(1+8 \eta_{2}\right) v_{2}^{4}}{8 v_{v}}, \\
a_{x^{2} y^{2}} & =\frac{v_{1}^{2} v_{2}\left(-4 v_{1} \chi\left(1+2 \eta_{1}\right)+3 v_{2}\right)}{4 v_{v}} .
\end{aligned}
$$

For a stack of horizontal homogeneous orthorhombic layers, $p_{x}$ and $p_{y}$ are constant; thus, we can compute an average (effective) $A_{k}$, which incorporates the influence of the medium properties along the whole raypath

$$
A_{k}=\frac{1}{Z} \sum_{i} a_{k}^{(i)} z^{(i)}
$$

where $k$ corresponds to one of the coefficients of equation C-3 $\left(k=\left\{0, x^{2}, y^{2}, x^{4}, y^{4}, x^{2} y^{2}\right\}\right), Z$ is the total depth, $a_{k}^{(i)}$ and $z^{(i)}$ are, respectively, the interval coefficient and the thickness of the $i$ th layer.

The objective of a Dix inversion is to obtain the interval parameters given the effective values. Thus, we compute expressions for the parameters $v_{v}, v_{1}, v_{2}, \eta_{1}, \eta_{2}$, and $\chi\left(\chi=\sqrt{1+2 \delta_{3}}\right)$ as a function of the coefficients $a_{0}, a_{x^{2}}, a_{y^{2}}, a_{x^{4}}, a_{y^{4}}$, and $a_{x^{2} y^{2}}$ using equations $\mathrm{C}-4$, and we obtain the following relations: 


$$
\begin{aligned}
& v_{v}=\frac{1}{a_{0}}, \\
& v_{1}=\sqrt{2 v_{v}\left|a_{x^{2}}\right|}, \\
& v_{2}=\sqrt{2 v_{v}\left|a_{y^{2}}\right|}, \\
& \eta_{1}=-\frac{v_{v} a_{x^{4}}}{v_{1}^{4}}-\frac{1}{8}, \\
& \eta_{2}=-\frac{v_{v} a_{y^{4}}}{v_{2}^{4}}-\frac{1}{8}, \\
& \chi=\frac{3 v_{1}^{2} v_{2}^{2}-4 v_{v} a_{x^{2} y^{2}}}{4\left(1+2 \eta_{1}\right) v_{1}^{3} v_{2}} .
\end{aligned}
$$

To obtain the interval parameters of a layered medium, we first compute the effective coefficients $A_{k}^{(i)}$ and $A_{k}^{(i-1)}$, using the relations C-4 and the effective model properties corresponding to two consecutive layers $i$ and $i-1$, and then we calculate the interval coefficients $a_{k}^{(i)}$ using the following relation:

$$
a_{k}^{(i)}=\left(A_{k}^{(i)} Z^{(i)}-A_{k}^{(i-1)} Z^{(i-1)}\right) / d z^{(i)},
$$

where $Z^{(i)}$ and $Z^{(i-1)}$ are the depth of the layers $i$ and $i-1$, respectively and $d z^{(i)}$ is the thickness of the $i$ th layer. Finally, we use equations C-6 to compute the interval parameters.

\section{REFERENCES}

Al-Dajani, A., I. Tsvankin, and M. Toksöz, 1998, Nonhyperbolic reflection moveout for azimuthally anisotropic media: 68th Annual International Meeting, SEG, Expanded Abstracts, 1479-1482.

Alkhalifah, T., 2003, An acoustic wave equation for orthorhombic anisotropy: Geophysics, 68, 1169-1172, doi: 10.1190/1.1598109.

Alkhalifah, T., 2011, Scanning anisotropy parameters in complex media: Geophysics, 76, no. 2, U13-U22, doi: 10.1190/1.3553015.

Alkhalifah, T., 2012, Traveltime approximations for transversely isotropic media with an inhomogeneous background: Geophysics, 77, no. 3, WA31-WA42, doi: 10.1190/geo2011-0465.1.

Alkhalifah, T., 2013, Traveltime approximations for inhomogeneous transversely isotropic media with a horizontal symmetry axis: Geophysical Prospecting, 61, 495-503, doi: 10.1111/j.1365-2478.2012.01067.x.

Alkhalifah, T., and I. Tsvankin, 1995, Velocity analysis for transversely isotropic media: Geophysics, 60, 1550-1566, doi: 10.1190/1.1443888.

Bakulin, A., V. Grechka, and I. Tsvankin, 2000, Estimation of fracture parameters from reflection seismic data. Part II: Fractured models with orthorhombic symmetry: Geophysics, 65, 1803-1817, doi: 10.1190/1.1444864

Bender, C. M., and S. A. Orszag, 1978, Advanced mathematical methods for scientists and engineers: McGraw-Hill.

Červený, V., 2001, Seismic ray theory: Cambridge University Press.

Grechka, V., and I. Tsvankin, 1999, 3-D moveout velocity analysis and parameter estimation for orthorhombic media: Geophysics, 64, 820-837, doi: 10 $.1190 / 1.1444593$

Hao, Q., and A. Stovas, 2016, Analytic calculation of phase and group velocities of P-waves in orthorhombic media: Geophysics, 81, no. 3 , C79-C97, doi: 10.1190/geo2015-0156.1.
Ibanez-Jacome, W., T. Alkhalifah, and U. Waheed, 2014, Effective orthorhombic anisotropic models for wavefield extrapolation: Geophysical Journal International, 198, 1653-1661, doi: 10.1093/gji/ggu229.

Kainkaryam, S., M. Decker, O. Zdraveva, J. Mathewson, C. Nalepa, R. Bachrach, R. Bloor, and D. Nichols, 2015, Ray based imaging and model building in tilted orthorhombic media: 77th Annual International Conference and Exhibition, EAGE, Extended Abstracts, We N114 11.

Ma, X., and T. Alkhalifah, 2013, $\mathrm{qP}$ wave traveltime computation by an iterative approach: 75th Annual International Conference and Exhibition, EAGE, Extended Abstracts, We 0209.

Masmoudi, N., and T. Alkhalifah, 2014, Multi-parameters scanning in HTI media: 84th Annual International Meeting, SEG, Expanded Abstracts, $480-485$

Masmoudi, N., and T. Alkhalifah, 2015a, Multi-parameter estimation for orthorhombic media: 85th Annual International Meeting, SEG, Expanded Abstracts, 504-508.

Masmoudi, N., and T. Alkhalifah, 2015b, Traveltime approximations for inhomogeneous orthorhombic media: 77th Annual International Conference and Exhibition, EAGE, Extended Abstracts, Tu N102 15.

Masmoudi, N., and I. Pšenčík, 2014, Approximate P-wave ray tracing and dynamic ray tracing in weakly orthorhombic media of varying symmetry orientation: 76th Annual International Conference and Exhibition, EAGE, Extended Abstracts, We G105 16.

Rawlinson, N., and M. Sambridge, 2004, Wave front evolution in strongly heterogeneous layered media using the fast marching method: Geophysical Journal International, 156, 631-647, doi: 10.1111/j.1365-246X.2004 .02153.x.

Schoenberg, M., and K. Helbig, 1997, Orthorhombic media: Modeling elastic wave behavior in a vertically fractured earth: Geophysics, 62, 1954 1974, doi: 10.1190/1.1444297.

Sethian, J. A., 1996, A fast marching level set method for monotonically advancing fronts: Proceedings of the National Academy of Sciences of the United States of America, 93, 1591-1595, doi: 10.1073/pnas.93.4.1591.

Sripanich, Y., and S. Fomel, 2015, On anelliptic approximations for qP velocities in transversely isotropic and orthorhombic media: Geophysics, 80, no. 5, C89-C105, doi: 10.1190/geo2014-0534.1.

Stovas, A., and T. Alkhalifah, 2012, A new traveltime approximation for TI media: Geophysics, 77, no. 4, C37-C42, doi: 10.1190/geo2011-0158.1.

Tsvankin, I., 1997, Anisotropic parameters and P-wave velocity for orthorhombic media: Geophysics, 62, 1292-1309, doi: 10.1190/1.1444231.

Tsvankin, I., J. Gaiser, V. Grechka, M. van der Baan, and L. Thomsen, 2010 Seismic anisotropy in exploration and reservoir characterization: An overview: Geophysics, 75, no. 5, 75A15-75A29, doi: 10.1190/1.3481775.

Tsvankin, I., and L. Thomsen, 1994, Nonhyperbolic reflection moveout in anisotropic media: Geophysics, 59, 1290-1304, doi: 10.1190/1.1443686. van Trier, J., and W. W. Symes, 1991, Upwind finite-difference calculation of traveltimes: Geophysics, 56, 812-821, doi: 10.1190/1.1443099.

Vasconcelos, I., and I. Tsvankin, 2006, Non-hyperbolic moveout inversion of wide-azimuth P-wave data for orthorhombic media: Geophysical Prospecting, 54, 535-552, doi: 10.1111/j.1365-2478.2006.00559.x.

Vidale, J. E., 1990, Finite-difference calculation of traveltimes in three dimensions: Geophysics, 55, 521-526, doi: 10.1190/1.1442863.

Waheed, U., and T. Alkhalifah, 2013, Efficient traveltime solutions of the TI acoustic eikonal equation: 75th Annual International Conference and Exhibition, EAGE, Extended Abstracts, Th 0611.

Waheed, U., T. Alkhalifah, and A. Stovas, 2013, Diffraction traveltime approximation for TI media with inhomogeneous background: Geophysics, 78, no. 5, WC103-WC11, doi: 10.1190/geo2012-0413.1.

Waheed, U., T. Alkhalifah, and H. Wang, 2015a, Efficient traveltime solution of the acoustic TI eikonal equation: Journal of Computational Physics, 282, 62-76, doi: 10.1016/j.jcp.2014.11.006.

Waheed, U., C. E. Yarman, and G. Flagg, 2015b, An iterative, fast-sweeping-based eikonal solver for 3D tilted anisotropic media: Geophysics, 80, no. 3, C49-C58, doi: 10.1190/geo2014-0375.1.

Wild, P., and S. Crampin, 1991, The range of effects of azimuthal isotropy and EDA anisotropy in sedimentary basins: Geophysical Journal International, 107, 513-529, doi: 10.1111/j.1365-246X.1991.tb01412.x.

Xu, X., I. Tsvankin, and A. Pech, 2005, Geometrical spreading of P-waves in horizontally layered, azimuthally anisotropic media: Geophysics, 70, no. 5, D43-D53, doi: 10.1190/1.2052467. 